\title{
Primary Nasopharyngeal Hodgkin Lymphoma
}

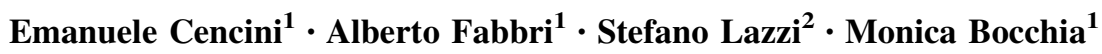

Received: 9 June 2015/ Accepted: 17 June 2015/Published online: 25 June 2015

(C) Springer Science+Business Media New York 2015

To the Editor,

We read with great interest the paper by Owosho et al. [1], showing a case of nasopharyngeal classical Hodgkin lymphoma (CHL) in a 49-year-old woman presenting with nasal congestion and post nasal drainage and we have a similar case managed at our institution.

CHL primary arising in the head and neck is a rare disease that represents approximately $1 \%$ of all CHL cases, and it is most frequent in young to middle-aged male patients. Only 35 cases have been identified to date and the most recent, homogeneous and large cohort reported in the literature comes from a retrospective analysis published by Iyengar and colleagues from the MD Anderson Cancer center, in which they showed that among more than 3.500 HL patients registered during a 40 years period (1967-2007), only 34 had a primary head-neck classic variant; notably nasopharynx localization is a true rarity since only 9 of them had an exclusive involvement at that level $[1,2]$. These epidemiologic data seem constant in time, since they are substantially in accordance with the ones previously reported by other authors [3-5]. Despite its rarity, primary nasopharynx $\mathrm{CHL}$ has a favourable clinical course and it is usually well managed with a combined approach, as reported by the authors and by Johnson and colleagues, too $[1,6]$. In particular, we share the opinion that the best therapeutic option in this setting is represented by few cycles of doxorubicin, bleomycin, vinblastine and dacarbazine

Emanuele Cencini

cencioema@libero.it

1 Unit of Hematology, Azienda Ospedaliera Universitaria Senese, University of Siena, Viale Bracci, 53100 Siena, Italy

2 Department of Human Pathology and Oncology, University of Siena, Siena, Italy
(ABVD) followed by attenuated dose involved-field radiotherapy (IF-RT), since we successfully treated 4 years ago a 50-year-old male patient with this modality. Of note, this patient presented bilateral nasal congestion and was diagnosed after 2 years of ineffective treatments for chronic rhinitis, including allergy medications and antibiotics. Histological diagnosis was, according to WHO classification [7], CHL (CD30 positive, CD15 positive in some cells, PAX5 positive with weak expression, MUM1 positive, OCT-2 and BOB.1 negative, LMP negative, EBER negative), with scattered Reed-Sternberg cells admixed with an inflammatory background. Computed tomography (CT) and positron emission tomography (PET) showed only nasopharyngeal localization, bone marrow biopsy was negative, Ann Arbor stage was IEA. The patient received two cycles of ABVD followed by IF-RT and achieved complete response (CR); he was alive and maintained $\mathrm{CR}$ at the last follow-up visit, 48 months following the end of therapy.

We would like to underline the importance, and sometimes the difficulty, to obtain a correct diagnosis, since the lesion may mimick both clinically and histologically a flogistic lesion like infectious mononucleosis or other lymphoid neoplasms such as NK-T cell lymphomas nasal type, T-cell/histiocyte-rich diffuse large B-cell lymphoma and EBV+ diffuse large B-cell lymphoma. Great caution is required before establishing a primary diagnosis of CHL in extranodal sites and subtype determination is often not possible. Therefore, immunohistochemical confirmation of the diagnosis is mandatory in primary extranodal CHL to rule out morphologic mimics.

In conclusion, stage IE is extremely rare in CHL and is characterized by an excellent outcome, as in our patient, that completed the therapeutic program without experiencing severe side effects, although he developed a permanent lower occipital alopecia after radiotherapy. 


\section{Compliance with Ethical Standards}

Conflict of interest The authors declare that they have no conflict of interest.

\section{References}

1. Owosho AA, Gooden CE, McBee AG. Hodgkin lymphoma of the nasopharynx: case report with review of the literature. Head Neck Pathol. 2015. doi:10.1007/s12105-015-0611-7.

2. Iyengar P, Mazloom A, Shihadeh F, Berjawi G, Dabaja B. Hodgkin lymphoma involving extranodal and nodal head and neck sites: characteristics and outcomes. Cancer. 2010;116:3825-9.
3. Aloulou S, Farhat H, Bosq J, Vanel D, Ribrag V, Turhan AG, et al. Hodgkin's disease primarily involving the oropharynx: case report and review of the literature. Hematol J. 2002;3:164-7.

4. Anselmo AP, Cavalieri E, Cardarelli L, Gianfelici V, Osti FM, Pescarmona E, et al. Hodgkin's disease of the nasopharynx: diagnostic and therapeutic approach with a review of the literature. Ann Hematol. 2002;81:514-6.

5. Bensouda Y, El Hassani K, Ismaili N, Lalya I, Boutayeb S, Benjaafar N, et al. Primary nasopharyngeal Hodgkin's disease: case report and literature review. J Med Case Rep. 2010;4:116.

6. Johnson ME, Robu VG, Turaka A. Nasopharyngeal Hodgkin lymphoma. J Clin Oncol. 2014;32:e40-1.

7. Swerdlow S, Campo E, Harris N, Jaffe E, Pileri S, Stein H, Thiele $\mathrm{J}$, Vardiman J. WHO classification of tumors of haematopoietic and lymphoid tissues. Lyon: IARC Press; 2008. 